\title{
Modeling of a Temperature Controller using Heat Exchangers
}

\author{
Aaron Don M. Africa, John Arvin A. Mercado, Joshua Kenichi S. Sim \\ Department of Electronics and Communications Engineering \\ De La Salle University, Manila \\ 2401 Taft Ave., Malate, Manila 1004, \\ Philippines, aaron.africa@dlsu.edu.ph
}

\begin{abstract}
Thermoregulators are devices or applications which control temperatures. Examples of such devices are air conditioners for the everyday use of people, or heat exchangers for use in industrial purposes like chemical power plants. These kinds of devices most of the time, if not all, require a feedback system for precise and accurate observation and monitoring of data and in turn maintain or manipulate the temperature to a more favorable value to lessen or mitigate damages and prevent such possible disasters. The paper aims to simulate a feedback-controlled heat exchanger system to lessen the errors done upon the physical execution. A program called MATLAB is required to show computations and calculations of possible outcomes with the user's inputs as a simulation with ideally precise outcomes. A program for calculation runs through MATLAB while the simulation is shown for monitoring and observing on SIMULINK.
\end{abstract}

Key words: MATLAB, temperature, feedback-controlled, simulation.

\section{INTRODUCTION}

With the advancements in technology, and with the rise of so many cities, factories, gadgets, and many more, temperature control has become a major part of making these possible. Whether it be making a room cold enough for a meeting, or cooling equipment in the factory to maintain its effectiveness or even to cooling a chip for the development of quantum computers using a nano-fridge as stated by [1], temperature control has everything to do with it. So many discoveries and so many automations required in one way or another the control of the environment that they are in, and the temperature is always and has always been one of the main factors that can affect any sort of process. Temperature makes almost everything react, from its effect on materials according to [2], to its effect on pressure, to its effect on conductivity, and many more. This means that controlling temperature can be one of the main things someone looks for when working in a sensitive environment. Having said this, almost all experiments, especially when it comes to the advancement in technology the control of temperature has become necessary and commonplace. The temperature has become a key component in making some things function optimally. Just like actual human bodies, as stated [3] human bodies regulate temperature on its own using several unique processes, there are also a numerous amount of systems in the world that require thermoregulation to function. Even in the simplest of day to day life, thermoregulation is seen on the regular. From a micro scale such as the cooling of someone's phone or personal computers as stated by [4], or to a macro scale such as cooling their cars, rooms, buildings, and many more, thermoregulation is present and seen. The regulation of temperature has also become important not only in the scene of technology but also in the world of art, and even the culinary world. Using a regulator to adjust and maintain a said temperature has done wonders in the preservation of numerous works of art that are one of a kind. The accuracy of temperature is also no less important, especially when it comes to cooking the ingredients the right way, such as beef which as stated by [5] has an optimal internal cooking temperature. After having stated everything, it is clear that the control of temperature has a vast amount of application in all aspects of the world, from the simple day to day life to even the most complicated and sensitive situations like the advancement of technology or the preservation of a work of art.

\section{BACKGROUND OF THE STUDY}

Thermoregulation is seen not only in the man-made world, but it is also present and observable in nature. The world has a natural way of regulating temperature, from the process of the water cycle as it brings forth rain as stated by [6], or how the ozone layer protects the earth from overheating by effectively blocking several rays from the sun as stated by [7], there is always a natural order of how the earth and nature cool itself and regulates the temperature on a localized or global scale. However, when it comes to this paper we will focus more on the man-made world, and how the regulation of temperature has had a massive impact on several fields of progress not just in science, but also in art. Just as how the most optimal things are seen in nature as the human eye, and how man tries to imitate the wonders of nature, this paper will focus on how temperature is controlled and optimized using a regulator that can detect a change in temperature and make adjustments accordingly to get the temperature back into the optimal state. 
This has several applications and can be very diverse when it comes to whatever field and level of expertise is needed. From a simple flick of a switch to turn on the air conditioner in a person's daily life, going to the accuracy needed when baking a cookie which according to [8] affects the sucrose and hexose that affects browning, or even the optimal temperature needed for automation of certain things for industrial work, temperature regulation is present. Taking a look at the industrial scene and how products are prepared and from start to finish, in almost every step of the process temperature is a key factor to consider. This paper focuses on a more macro scale of temperature control which as stated [9] also affects energy savings by controlling the temperature of a room or a factory where delicate processes are happening.

\section{STATEMENT OF THE PROBLEM}

Nowadays, even with advancements in so much technology, temperature control remains a problem. People wake up in the middle of the night with their rooms being too cold or hot due to the effect the temperature has with the quality of a person's sleep according to [10]. People also line up in the elevator only to be greeted by an elevator with no temperature regulator. Factories don't operate optimally due to the inconsistency of temperature, and so many more issues with temperature are arising every day. With the rise of global issues like climate change and global constant temperature, some equipment needs an optimal temperature, and even humans have an optimal working temperature [11]. This means that systems that rely on a constant source of temperature to function would need a regulator that adjusts accordingly and quickly. They would need a system that can detect changes in temperature, and either heat or cool the place accordingly to maintain said temperature.

\section{SIGNIFICANCE OF THE STUDY}

The ability to control and regulate the temperature on a macro and micro scale is relevant in almost all fields of life. People from any field or industry, anywhere in the world can appreciate and see the effects of thermoregulation on a day to day basis. When air conditioners became commercially available, and when the control of temperature became more and more common, it brought about convenience to the masses and people. What people don't see however is the impact of temperature control when it comes to every product that they buy. Whether that be a food product or an appliance, there is always a factory from where these things are mass-produced. In these set factories, temperature regulation is invaluable since almost every process requires a certain temperature to function optimally if not function at all as stated by [12]. With every breakthrough in technology, especially today almost always means experimentation, and in experimentation, there are variables to consider. These variables need to be controlled and regulated to accurately observe the results, and one of the key factors in these experiments is almost always temperature. Some examples being temperature sensitive rice in genetic outcrossing as stated by [13], performance optimization of thermoelectric devices in cooling computer chips according to [14], and even the cooling of a dual-processor computer as stated by [15]. To further progress and reach advancements in technology, to preserve the artifacts and history of the past, and even to maintain the present as industries mass produce products for the masses thermoregulation will always be present.

\section{DESCRIPTION OF THE SYSTEM}

The system requires the user input values so that the step changes in the system are monitored after showing the calculations. The values then will be handed over to the SIMULINK to show the user a program interface where the control type can be chosen from feedforward to feedback and the graph between the relation of both delay and advancing will be shown.

\section{METHODOLOGY}

The script was made to measure the step response in the steam valve voltage. Once that is done, The experimental and simulated response are to be observed and compared with each other and be shown the step response in the temperature setpoint. Once all factors are considered, the program will then check for the effect of disturbance in inflow temperature. Figure 1 shows the block diagram of the heat exchanger temperature control.

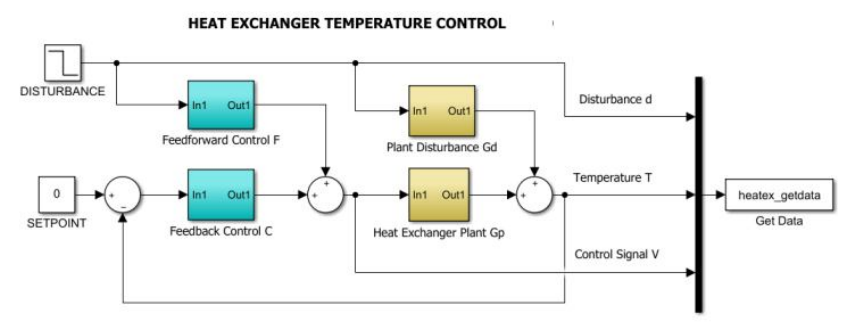

Figure 1: Heat exchanger temperature control

\section{REVIEW OF RELATED LITERATURE}

A research made [16], aims to make an automated temperature control of fluids using a heat exchanger with the functions of a heater that uses electrical resistance. The function of the system is recirculating the fluid inside a container that uses a control algorithm of on and offs. The system uses a programmable microcontroller wherein the microcontroller relies on the sensors for better and accurate temperature control [17]. The microcontroller also exhibits more features such as monitoring of the light and temperature for more reliable results and output.

According to [18], a way of controlling temperature using the heat exchanger is making use of the flue gas as a heating medium which is then combusted by solid fuel. The reactions happening in the medium is an efficient way of producing and spreading heat [19]. The heated medium is what they call a 
power gas, a mixture of air and water steam, and is led to a gas turbine that controls or drives the asynchronous generator. While heating is one issue, overheating is another. The study aims to make a preventive measure for further mitigation of disaster or damage made in the heat exchanger by measuring the main heat exchanger and evaluate the temperature which in turn will help the system not overheat by using the protective control feature of the said system.

In [20], a ventilation system, very much like an air conditioner when considering its function, is automated and functional based on the user's demand and the temperature in both indoors and outdoors. The development of the push-pull ventilation system is to maximize and be energy efficient in the operation of ventilation performance [21]. The principle used in the push-pull ventilation is by putting up a wall for the opening and closing of the air intake that controls the temperature. The system is most useful in areas that require comfortable and permissible temperature such as houses and rooms that have delicate materials to be in.

In [22] a modern control systems simulation on MATLAB state that the use of the said program, MATLAB, is almost, if not the same, similar to the laboratory trials and simulations when it comes to feedback linearization techniques. The usage of MATLAB in getting an idea of the feedback linearization outcome is of quality of life to the user since the program can be run locally whenever a certain device, such as computers and laptops, have the program are installed are present. If the former devices are not available, the program could also be running through the internet using smartphones and laptops that do not have the program installed in it [23].

The paper of [24] states that humidity plays a huge factor in air conditioning and is a great way to make a difference in both thermal comfort and indoor air quality. It is stated that small to medium-sized buildings almost have no device that can help in the monitoring of moisture due to limitations and can leave humidity without regulation. Using air conditioning systems, the goal is to eliminate moisture and humidity using control loops. Control algorithms are also used and developed to save energy and have better control performance once each of the devices is embedded with each other. The very common feedback and control system as stated in the article is that there are two coils mainly for air conditioning. The outside air, which acts as the variable for whether the air should be conditioned to be colder or not is present. It will then go past the sensor whether the temperature is well set and will supply the needed temperature of the air as time passes by using a heat exchange network. Their main concern is that the loss of power in chemical plants that rely on computers.

\section{THEORETICAL CONSIDERATION}

Considering that an expert system is meant to maximize ease of use regardless of its user, it may be assumed that an interface that is straightforward and easily understood is considered and implemented in the designed system. The program is designed to simply take note of the changes in temperature and monitor the said changes in the experiment. The interface of the MATLAB program [25], due to its nature of being more of a calculator than an actual executable program file, seems bland and simple. The function of the program however, no matter how aesthetically unpleasing it may seem, will always be up to its expected performance.

\section{DATA AND RESULTS}

Figures 2 to 7 shows the output of the simulation using Matlab.

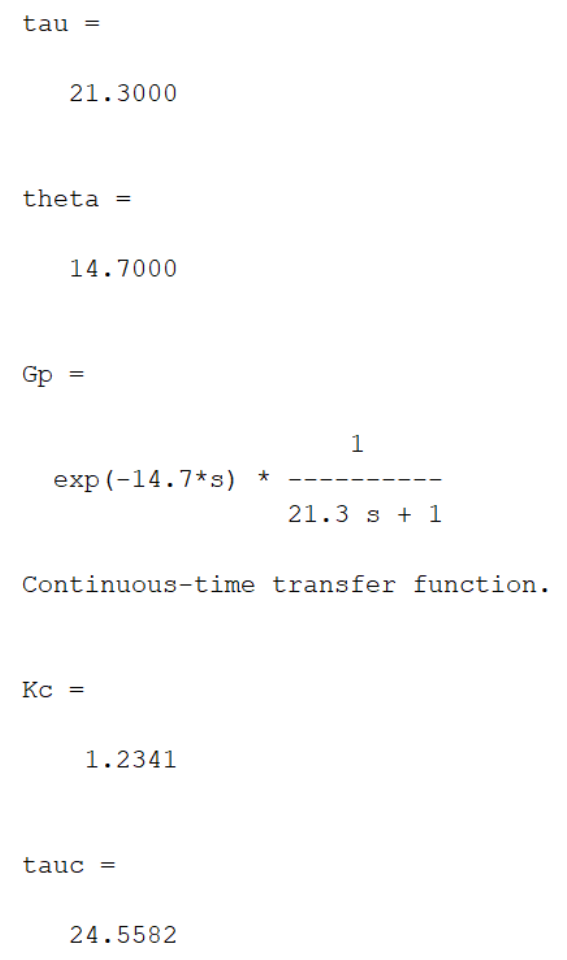

Figure 2: Initial MATLAB Simulation
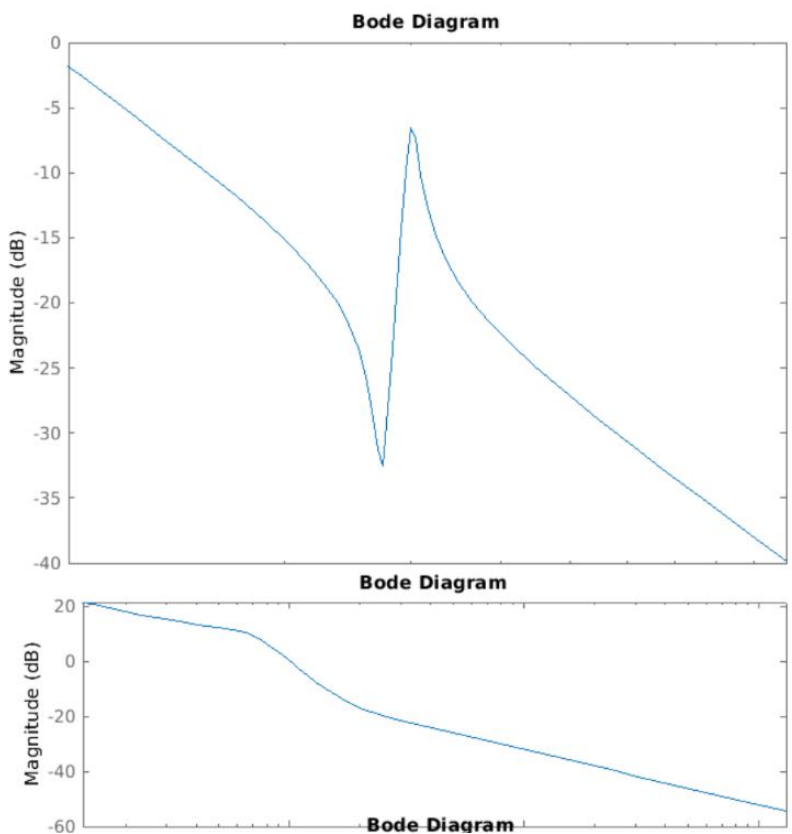

Figure 3: The Bode Plot of the Heat Exchanger's Feedback 


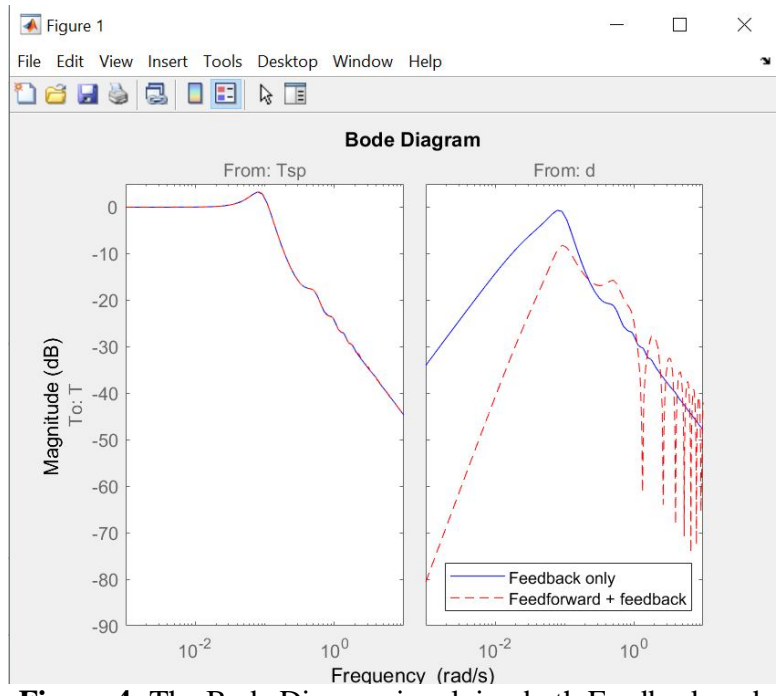

Figure 4: The Bode Diagram involving both Feedback and Feedforward

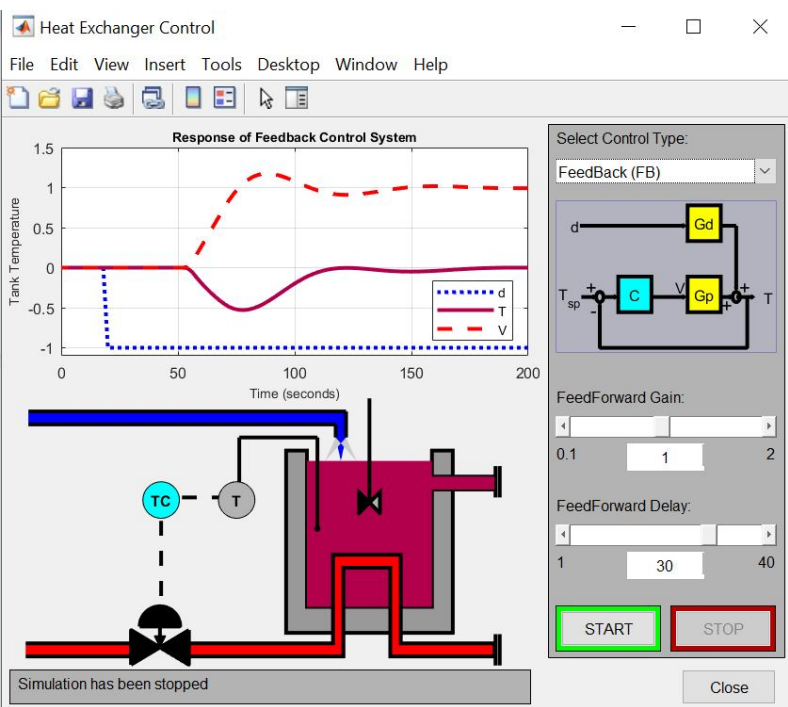

Figure 5: The interface of the program made for simulating in MATLAB in a Feedback Control Type

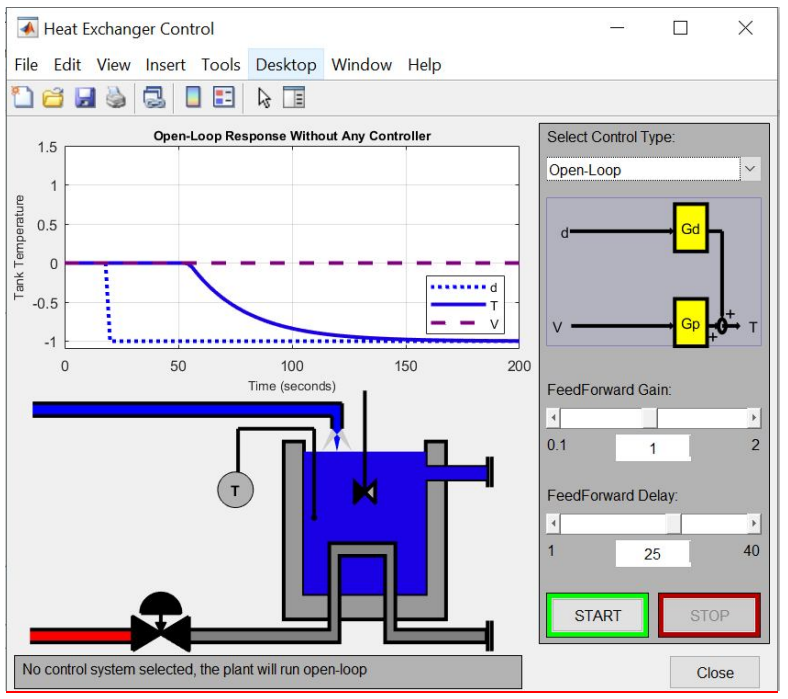

Figure 6: The interface of the program with an Open-Loop Control Type

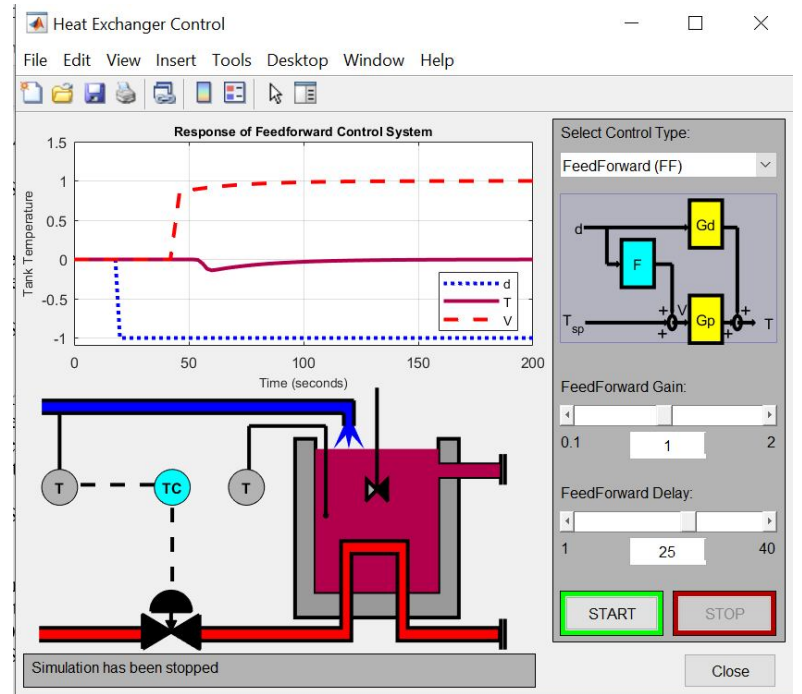

Figure 7: The interface of the simulation with a Feedforward Control Type

\section{ANALYSIS OF DATA}

The first part of the program asks for the user's input is asking for the measured response to a step change in the voltage of the steam valve. First, the value of tau will be computed for in the process. The value that came out for tau is 21.3. The tau is needed for computing the other values such as the theta value which came out to be 14.7. The values are then used for experimented and are observed by getting to know the tank temperature. The Gp value is also being able to be seen as the theta and tau have been computed and have been fed in the equations.

The magnitude, in decibels, delivered by the system is gradually becoming lower about longer frequencies. From a valued given by the user, the bode diagram plot shows the graph with feedback and feedforward is exhibiting almost the same results with each other meaning that the system has gone through a successful temperature change.

The simulations show how the heat exchanger behaves while controlling the temperature of the said system with different kinds of control types. The Open-Loop Control type is effective in cooling the system off, while the feedback and feedforward control types are very good at maintaining temperatures. The only difference between both outcomes is that the feedforward function is proven to be the most effective when maintaining temperatures.

\section{CONCLUSION}

Seeing the data and results, and after analyzing the data that was given by the MATLAB simulation, we can see that the system works as it should. All the variables connect and make sense, the response time is not long. The feedback from the sensors is also seen to be accurate since the temperature is controlled and regulated just fine and can be kept there without a problem. It is therefore concluded and see that the system is an effective way to be regulating the temperature in an environment that is suitable. The system can function to be 
even more effective and efficient by calculating and readjusting the circuit of the sensors to produce an output that can be even more reliable and stable. This would also reduce the response time that it would take for the system to adjust to the said input that is needed, therefore achieving the desired output in an even shorter period.

\section{RECOMMENDATIONS}

After careful consideration, and after analyzing the data given by MATLAB on the simulation of the system when it comes to thermal regulation it is recommended that further research be done when it comes to the accuracy and response time of the sensors. Further optimization can be attained by calculating and readjusting the circuit of the sensors to produce an output that can be even more reliable and stable. Another thing that might come into consideration it how large or small the venue that the system needs to function upon. With the current projected system, it is recommended that it be used in a larger scale, such as factories and buildings that do not require a very accurate and stable hold on the temperature and where the response time can be more forgivable when it comes to its effects on the output. This can be applied in situations such as a production line where the product needs to be kept at a certain temperature to preserve quality and consistency. When it comes to the effectiveness and the accuracy of the output that the system gives it is recommended that it not be used in situations that have a very sensitive process that is affected heavily by the cooling and heating of variables such as experimentation. Something that can also be further researched upon is other ways of cooling and heating aside from the processes stated in the project, perhaps something more sustainable and that can be recycled. The lack of an actual simulation or experiment is compensated by a MATLAB simulation and program that runs on a computer projecting the outcome that is expected. However, in the real world, several factors and variables cannot be seen or controlled therefore giving off a higher percentage for error. It is recommended that an actual simulation or experiment be conducted to complement the simulation given by the MATLAB software for an even more accurate prediction of how the system can work and how effective it can be in different situations and environments.

\section{REFERENCES}

[1] J. Ouellette, "Nanofridge keeps quantum computers cool," New Scientist. Vol. 234,No. 3125, p. 11, 2017. https://doi.org/10.1016/S0262-4079(17)30909-0

[2] H. Zhang, S. Kim, G. Choi, D. Xie and H. Cho, "Effect of temperature dependent material properties on thermoelastic damping in thin beams," International Journal of Heat and Mass Transfer. Vol. 139, pp.1031-1036, 2019.

[3] Z. Kang, F. Wangand Udayraj, "An advanced three-dimensional thermoregulation model of the human body: Development and validation," International Communications in Heat and Mass Transfer. Vol. 107, pp. 34-43, 2019.
[4] R. Grimes, E. Walsh and P. Walsh, "Active cooling of a mobile phone handset," Applied Thermal Engineering. Vol. 30,No. 16, pp. 2363-2369, 2010.

[5] M.López Osornio, G.Hough, A.Salvador, E.ChambersIV, S.McGraw and S.Fiszman, "Beef's optimum internal cooking temperature as seen by consumers from different countries using survival analysis statistics,"Food Quality and Preference. Vol.19, No. 1, pp.12-20, 2008. https://doi.org/10.1016/j.foodqual.2007.06.004

[6] S. Zhang, M.Xiang, Z. Xu, L. Wang and C. Zhang, "Evaluation of water cycle health status based on a cloud model,'Journal of Cleaner Production. Vol. 245, 2020.

[7] A.K.Singh and A. Bhargawa, "Atmospheric burden of ozone depleting substances (ODSs) and forecasting ozone layer recovery," Atmospheric Pollution Research. Vol. 10, No. 3, pp. 802-807, 2019. https://doi.org/10.1016/j.apr.2018.12.008

[8] L. Ameur, O. Mathieu, V. Lalanne, G. Trystram and I. Birlouez-Aragon, "Comparison of the effects of sucrose and hexose on furfural formation and browning in cookies baked at different temperatures,"Food Chemistry. Vol. 101, No. 4, pp. 1407-1416, 2007.

[9] L.Lan, Z.W.Lian, X.L.Qian and C.Z. Dai, "The effects of programmed air temperature changes on sleep quality and energy saving in bedroom,"Energy and Buildings. Vol. 129, pp. 207-214, 2016. https://doi.org/10.1016/j.enbuild.2016.08.001

[10]S. Joshi, T. Lesser,J.Olsen and B. O'Hara, "The importance of temperature and thermoregulation for optimal human sleep," Energy and Buildings. Vol. 131, pp. 153-157, 2016.

[11]E. Julianto, W. Siswanto and M. Effendy, "Characteristics of temperature changes and stress of float glass under heat radiation," International Journal of Emerging Trends in Engineering Research. Vol. 7, No. 9, pp.228-233, 2019. https://doi.org/10.30534/ijeter/2019/03792019

[12] D. Mourtzis, D. Tsakalos, F. Xanthi and V. Zogopoulos, "Optimization of highly automated production line: An advanced engineering educational approach," Procedia Manufacturing. Vol. 31, pp. 45-51, 2019.

[13] S. Arasakesary, S. Manonmani, R. Pushpam and S. Robin, "New Temperature Sensitive Genic Male Sterile Lines with Better Outcrossing Ability for Production of Two-Line Hybrid Rice," Rice Science.Vol. 22, No. 1, pp. 49-52, 2015. https://doi.org/10.1016/j.rsci.2015.05.011

[14] H. Saber, S. AlShehri and W. Maref, "Performance optimization of cascaded and non-cascaded thermoelectric devices for cooling computer chips," Energy Conversion and Management. Vol. 191, pp. 174-192, 2019.

[15] S.Wiriyasart, C. Hommalee and P. Naphon, "Thermal cooling enhancement of dual processors computer with thermoelectric air cooler module," Case Studies in Thermal Engineering. Vol. 14, 2019.

[16] M. Dulău, M. Karoly and T. Dulău, "Fluid temperature control using heat exchanger," Procedia Manufacturing. Vol. 22, pp. 498-505, 2018. 
Aaron Don M. Africa et al., International Journal of Emerging Trends in Engineering Research, 8(7), July 2020, 3185 - 3190

https://doi.org/10.1016/j.promfg.2018.03.058

[17] A. Africa, A. Divino and K. Hartigan-Go, "Fuzzy logic temperature control: A feedback control system implemented by fuzzy logic," International Journal of Emerging Trends in Engineering Research. Vol. 8, No. 5, pp. 1879-1885, 2020.

+https://doi.org/10.30534/ijeter/2020/66852020

[18] B. Filipova, P. Nevriva and M. Pies, "The Temperature Time Responses of the Heat Exchanger Equipped by the Protective Control," IFAC-PapersOnLine. Vol. 49, No. 25, pp. 487-492, 2016.

[19] B. Asgari, M. Habibi and A. Hakkaki-Fard, “Assessment and comparison of different arrangements of horizontal ground heat exchangers for high energy required applications," Applied Thermal Engineering. Vol. 167, 2020.

[20]A. Fukushimaand S. Murata, "Push-pull Mechanical Ventilation System with Heat Exchanger Controlled by Intake Air Temperature," Energy Procedia. Vol. 78, pp. 43-48, 2015.

https://doi.org/10.1016/j.egypro.2015.11.112

[21] B. Zhou, F. Chen, Z. Dong and P. Nielsen, "Study on pollution control in residential kitchen based on the push-pull ventilation system," Building and Environment. Vol. 107, pp. 99-112, 2016.

[22]P.Bisták, M.Halás and M.Huba, "Modern Control Systems via Virtual and Remote Laboratory Based on Matlab," IFAC-PapersOnLine. Vol. 50, No. 1, pp. 13498-13503, 2017.

[23] P.Bistak and M.Huba, "Three-Tank Virtual Laboratory for Input Saturation Control Based on Matlab," IFAC-PapersOnLine. Vol. 49, No. 6, pp. 207-212, 2016. https://doi.org/10.1016/j.ifacol.2016.07.178

[24] W. Chen, G. Fang, C. Wang, W. Weng, M. Chan, S. Deng, X. Liu and H. Yan, "An experimental study on a novel direct expansion based temperature and humidity independent control air conditioning system," Energy Procedia. Vol. 158, pp. 2237-2243, 2019.

https://doi.org/10.1016/j.egypro.2019.01.172

[25] Matlab. https://www.mathworks.com/products/matlab.html. 2020 . 